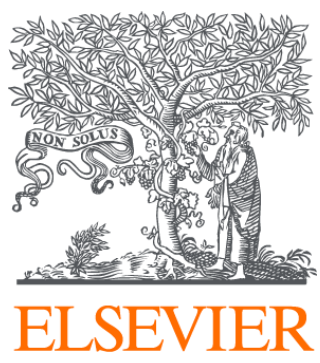

Since January 2020 Elsevier has created a COVID-19 resource centre with free information in English and Mandarin on the novel coronavirus COVID-

19. The COVID-19 resource centre is hosted on Elsevier Connect, the company's public news and information website.

Elsevier hereby grants permission to make all its COVID-19-related research that is available on the COVID-19 resource centre - including this research content - immediately available in PubMed Central and other publicly funded repositories, such as the WHO COVID database with rights for unrestricted research re-use and analyses in any form or by any means with acknowledgement of the original source. These permissions are granted for free by Elsevier for as long as the COVID-19 resource centre remains active. 


\title{
Sensory-processing sensitivity and COVID-19 stress in a young population: The mediating role of resilience
}

\author{
Shuhei Iimura \\ The University of Tokyo, Japan \\ Japan Society for the Promotion of Science, Japan
}

\section{A R T I C L E I N F O}

\section{Keywords:}

Sensory-processing sensitivity

Environmental sensitivity

Resilience

COVID-19

Stress

Adolescence

Young adulthood

\begin{abstract}
A B S T R A C T
Psychologists worldwide are becoming increasingly concerned about the negative impact of the novel coronavirus (COVID-19) pandemic on adolescents' mental health. However, compared to studies involving adults, research using a young population is limited. To further understand the mental health of older adolescents and young adults during the pandemic, the present study examined whether resilience, as a protective factor, buffers the relationship between the personality trait of environmental sensitivity and COVID-19-related distress. In total, 441 older adolescents and young adults (53.7\% women, $M_{\text {age }}=18.91$ years, $S D_{\text {age }}=0.82$ years) living in urban Japan completed an online cross-sectional survey in October 2020. The results showed that sensitivity was positively, though weakly, correlated with COVID-19 stress and negatively correlated with resilience. Resilience was negatively correlated with COVID-19 stress. Mediation analysis showed that resilience buffered the negative relationship between sensitivity and COVID-19 stress, and its indirect effect was statistically significant, albeit close to zero. These results suggest that higher sensitivity is not necessarily a vulnerability factor, if resilience can be enhanced.
\end{abstract}

\section{Introduction}

As of 2019, mankind has been faced with the global spread of the novel coronavirus (COVID-19). As of April 2021, more than $129,000,000$ people have been infected with COVID-19 worldwide, with more than 2,800,000 deaths (John Hopkins University, 2020; World Health Organization [WHO], 2021). Each country is implementing measures, such as lockdown and school closure, to help control the spread of COVID-19, as outbreaks not only impair physical health, but also significantly impact mental health. In fact, many studies have reported increased psychopathology amidst the COVID-19 pandemic, including anxiety, depression, traumatic stress, coronary phobia, and xenophobia (e.g., Magson et al., 2021; Mertens et al., 2020; Qi et al., 2020; Tang et al., 2021; Taylor et al., 2020; van der Velden et al., 2020; Xiong et al., 2020; Zhang et al., 2020). Many studies also point to the importance of interventions and evidence-based policy decisions on the part of health care practitioners to prevent or reduce the negative consequences of the COVID-19 pandemic. Considering these social situations and research trends, this study explores the personality traits that make people more susceptible to negative influences amidst the COVID19 pandemic, as well as factors that may be protective.

\subsection{Mental health during the COVID-19 pandemic}

Although there is a limited number of related studies on adolescents and young adults (i.e., 10-24 years; Sawyer et al., 2018) compared to research on the older population, studies from around the world suggest that the youth are more likely to experience mental health problems during the COVID-19 pandemic due to school closure and reduced family well-being (e.g., Golberstein et al., 2020; Prime et al., 2020). In fact, a recent systematic review reported that membership of a younger age group ( $<40$ years) or being a student (Xiong et al., 2020) are risk factors associated with COVID-19 distress. For example, Magson et al. (2021) demonstrated that from the pre- to pandemic period, Australian adolescents experienced increased depressive symptoms and anxiety, and decreased life satisfaction, which were particularly pronounced among girls. They also showed that increased COVID-19-related worries, online learning difficulties, and conflicts with parents predicted poorer mental health. Using a sample of Chinese adolescents, Duan et al. (2020) showed that smartphone and Internet addiction were positively associated with depression and that the tendency to use problem-focused coping styles negatively explained depression. In addition, according to Tang et al. (2021), senior secondary school

\footnotetext{
* The University of Tokyo, 7-3-1 Hongo Bunkyo-ku, Tokyo 113-0033, Japan.

E-mail address: iimurashuhei@gmail.com.
} 
students in China had higher levels of psychopathology and lower levels of life satisfaction than primary school students. Perceived benefits of home quarantine and parent-child discussions about COVID-19 were negatively correlated with psychopathology and positively correlated with life satisfaction. Moreover, Qi et al. (2020) found that Chinese adolescents who perceived themselves as having low social support reported higher depressive and anxiety symptoms, even after controlling for the effects of gender, grade, and degree of COVID-19 exposure.

In summary, adolescents' poor mental health during the COVID-19 pandemic, as described above, may be due to a variety of complex factors, including increased lockdown-induced social distance (e.g., from friendships, which are an important resource in adolescence), economic recession, school closure, and reduced family well-being (Golberstein et al., 2020; Prime et al., 2020).

\subsection{Individual differences in environmental sensitivity}

Sensitivity to environmental influences varies among people (Pluess, 2015). In other words, highly sensitive people are more likely to experience adversarial effects in stressful environments, such as the COVID19 pandemic. Individual differences in sensitivity can be captured by the concept of Environmental Sensitivity or its dispositional aspect, sensoryprocessing sensitivity, which is defined as the ability to process and register both positive and negative environmental influences or experiences (Greven et al., 2019; Pluess, 2015).

Due to their traits, highly sensitive young people are more likely to experience poor mental health in negative environments. For example, recent evidence has shown that compared to less sensitive adolescents, highly sensitive adolescents experience lower socio-emotional wellbeing when the impact of negative life events experienced in the preceding week was significant (Iimura, 2021). Moreover, several crosssectional studies have reported that high sensory-processing sensitivity in adolescents is positively correlated with depressive symptoms and anxiety (Liss et al., 2005; Liss et al., 2008).

Also due to their characteristics, highly sensitive adolescents are more likely to benefit from supportive environments. Studies have also reported that in supportive school environments, highly sensitive adolescents exhibit higher socio-emotional well-being than those with lower sensitivity (Iimura \& Kibe, 2020).

Could sensitivity be a vulnerability factor during the COVID-19 pandemic? Perhaps the answer is that youth mental health can be moderated, for better or for worse, by protective factors and/or a supportive environment. According to the theory of Environmental Sensitivity (Greven et al., 2019; Pluess, 2015), highly sensitive youth would show a greater decline in socio-emotional well-being during a pandemic if the environmental quality is less supportive, and they would show no decline in mental health in the presence of more supportive factors.

\subsection{The protective role of resilience against COVID-19 distress}

Resilience is one of the most widely discussed protective factors against adversity and traumatic stress. Resilience can be defined in a variety of ways, but its central idea is to bounce back or recover from stress (Smith et al., 2008). Recently, researchers have pointed out the importance of resilience during the COVID-19 pandemic (Chen \& Bonanno, 2020; Prime et al., 2020).

Although they are quite limited in number, some studies have revealed the characteristics of resilient people during the COVID-19 pandemic. For example, Killgore et al. (2020) showed that the mean score for resilience was lower than in the pre-pandemic period, but that people who tend to go outside more often, exercise more, perceive a higher degree of social support from family, friends, and significant others, and sleep better were more resilient. In addition, Verdolini et al. (2021) reported that for patients with mental illness, pursuing hobbies and structure in the family environment were associated with higher levels of resilience. Furthermore, Zager Kocjan et al. (2021) indicated that resilience buffers the negative association between the Big Five of neuroticism and COVID-19 stress.

It is important to note that the evidence reviewed above suggests that resilience amidst a pandemic is associated with modifiable factors. Thus, individual resilience can be enhanced. For example, recent research has demonstrated that highly sensitive adolescents respond well to resilience-enhancing psychoeducational interventions; i.e., they experienced less depression and higher self-efficacy (Kibe et al., 2020; Pluess \& Boniwell, 2015). Interventions to increase resilience during the COVID-19 pandemic may be beneficial to improve youth mental health.

\subsection{The current study}

As noted thus far, individual differences in sensory-processing sensitivity (i.e., susceptibility) and resilience (i.e., protective factors) may be important elements in further understanding youth mental health during the COVID-19 pandemic. Nonetheless, our knowledge of these factors' role is limited. Therefore, the current study aimed to examine how sensory-processing sensitivity and resilience in youth aged 18-24 years old are related to COVID-19-induced distress. This study examined the hypothetical model shown in Fig. 1 to investigate the mediating effect of resilience. The current study predicted that sensoryprocessing sensitivity would be positively associated with COVID-19 stress, but that the association would be buffered by resilience.

\section{Method}

\subsection{Procedure and participants}

The first author invited students enrolled in a course on selfunderstanding $(n=588)$ at a university in Tokyo to participate in an online cross-sectional survey. Students who expressed interest were provided with a link to complete the survey. The survey using Google Forms was administered on October 28, 2020, during the COVID-19 pandemic. Informed consent was obtained virtually on the first page of the online survey. Participation in the study was voluntary, and participants did not receive any compensation. This study was approved by the ethics review committee of the first author's institution (No. 20374).

Although 450 students completed the survey (response rate $=$ $76.5 \%$ ), nine students' data were removed, as their responses were

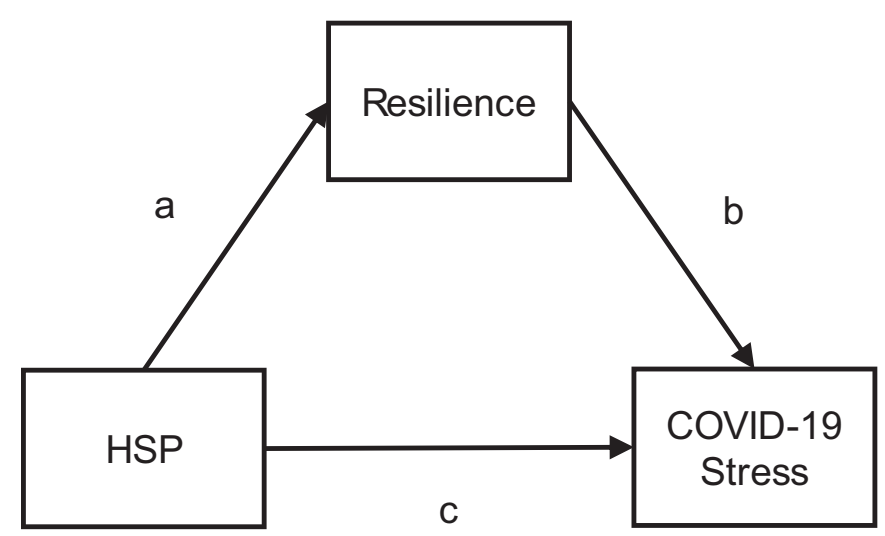

(c')

Fig. 1. Hypothetical mediation model note. Although this model controls for the effects of gender and age, these paths are drawn in abbreviated form. The coefficient "a" represents the partial regression coefficient between HSP and resilience; the coefficient "b" between resilience and COVID-19 Stress; and the coefficient "c" between HSP and COVID-19 Stress when the mediator is added. The coefficient " $\mathrm{c}$ " "refers to the coefficient in the model without the mediator. HSP $=$ sensory-processing sensitivity. 
inadequate (i.e., participants who were neither youth nor Japanese). Data from 441 Japanese youth were used in the analysis. Of the participants, 237 (53.7\%) were women. The participants' mean age was 18.91 years $(S D=0.82, M I N=18.00, M A X=24.00)$. Hence, all study participants were in late adolescence and young adulthood (Sawyer et al., 2018), which is suited to the study's purpose. No missing values were included in the dataset.

\subsection{Measures}

The 10-item Japanese version of the Highly Sensitive Person Scale (Iimura et al., 2021) was used to measure individual differences in sensory-processing sensitivity. This scale consists of ten items that investigate sensitivity to positive environments (e.g., "Are you deeply moved by the arts or music?") and negative environments (e.g., "Are you easily overwhelmed by strong sensory input?"). Each item was rated on a 7-point Likert-type scale, ranging from 1 (strongly disagree) to 7 (strongly agree). The items' internal consistency was good, with Cronbach's $\alpha=0.85$.

One of the most widely used resilience scales, the Japanese version of the Brief Resilience Scale (Iimura \& Taku, 2018; Tokuyoshi \& Moriya, 2014), was applied to measure resilience. This scale has been used in several studies to measure resilience during the COVID-19 pandemic (e. g., Verdolini et al., 2021; Zhang et al., 2020). The scale consists of six items (e.g., "I tend to bounce back quickly after hard times" and "It does not take me long to recover from a stressful event"). Each item was rated on a 5 -point Likert-type scale, ranging from 1 (strongly disagree) to 5 (strongly agree). Internal consistency was good, with Cronbach's $\alpha=$ 0.85 .

The Japanese version of the COVID Stress Scale (Muta et al., 2020) was used to measure COVID-19-related distress. Participants completed this scale, reporting their level of COVID-19-related distress in the last 7 days. This scale consists of 36 items (e.g., "I am worried about contracting the virus" and "I am worried about keeping my family safe from the virus") and has five subscales: fear of danger and contamination, fear of economic consequences, xenophobia, compulsive checking and reassurance seeking, and traumatic stress symptoms related to COVID19. Overall scores were used in this study. Each item was rated on a 5point Likert-type scale, ranging from 0 (not at all) to 4 (extremely). The items' internal consistency was good, with Cronbach's $\alpha=0.93$.

\subsection{Data analysis}

Analysis in this study consisted of two sections. (1) First is a preliminary analysis. This section aims to understand the participants' basic characteristics; here, descriptive statistics and correlation coefficients for each variable were calculated. In addition, we tested for gender differences in each variable's mean. (2) The second section is a mediation analysis (see MacKinnon et al., 2007 for details). This study analyzed the relationship between sensory-processing sensitivity, resilience (i.e., the mediator), and COVID-19 stress by examining the hypothetical model depicted in Fig. 1. Goodness of fit was evaluated based on the following three indices: comparative fit index (CFI), root mean square error of approximation (RMSEA), and standardized root mean square residual (SRMR). Specifically, acceptable fit was indicated by CFI $\geq 0.90$, RMSEA $\leq 0.08$, and SRMR $\leq 0.80$ (e.g., Hu \& Bentler, 1999; Schermelleh-Engel et al., 2003). To test the indirect effect (i.e., a $\times$ b), a bootstrap analysis (5000 resamples) was conducted; if the upper and lower limits of the $95 \%$ confidence interval (CI) of the regression coefficient produced by the bootstrapping method do not cross 0 , the indirect effect is statistically significant. All analyses in this study were performed using R version 3.6.3 (R Core Team, 2020). The significance level was set at $5 \%$.

\section{Results}

\subsection{Preliminary analysis}

The means and standard deviations for sensory-processing sensitivity, resilience, and COVID-19 stress are shown in Table 1. Sensoryprocessing sensitivity was negatively correlated with resilience $(r=$ $-0.48,95 \%$ CI $[-0.54,-0.40], p<.001)$ and positively correlated with COVID-19 stress ( $r=0.25,95 \%$ CI $[0.16,0.34], p<.001)$. In addition, resilience was negatively correlated with COVID-19 stress $(r=-0.23$, $95 \%$ CI $[-0.31,-0.14], p<.001)$. Regarding these results, statistical power analysis, conducted using G*Power (Faul et al., 2009), confirmed that the current sample size had sufficient statistical power (power [1- $\beta$ ] $=0.999$ ) to detect $r>|0.23|$. A scatter plot depicting the relationship between these variables can be found in Supplementary materials Figs. S1-S3 (https://bit.ly/39gMjar).

The $t$-test showed that the mean value for sensory-processing sensitivity was higher in women $(M=4.66)$ than in men $(M=4.27)(t(439)$ $=3.72$, Cohen's $d=0.36, p<.001)$. The mean value for COVID-19 stress was also higher in women $(M=0.92)$ than in men $(M=1.05)(t(439)=$ 2.64 , Cohen's $d=0.25, p=.009$ ). There was sufficient statistical power to detect the former, $d=0.36$ (power $[1-\beta]=0.964$ ), but statistical power was somewhat weak with regard to detecting the latter, $d=0.25$ (power $[1-\beta]=0.743$ ).

\subsection{Mediation analysis}

The goodness of fit for the mediation model shown in Fig. 2 was excellent (CFI $=1.00$, RMSEA $=0.00$, SRMR $=0.00$ ). In the model that did not include the mediator (CFI $=0.96, \mathrm{RMSEA}=0.04, \mathrm{SRMR}=0.03$ ), sensory-processing sensitivity was positively associated with COVID-19 stress $\left(c^{\prime}=0.25, S E=0.02, p<.001\right)$. Similarly, in the model that included the mediator, the higher the sensitivity, the higher the COVID19 stress level ( $c=0.17, S E=0.03, p=.002$ ). Sensory-processing sensitivity was negatively related to resilience ( $\mathrm{a}=-0.47, S E=0.03$, $p<.001$ ), and resilience was negatively associated with COVID-19 stress (b $=-0.14, S E=0.03, p=.010$ ). Bootstrap analysis showed that the indirect effect of path $\mathrm{a} \times \mathrm{b}$ was statistically significant $(\mathrm{a} \times \mathrm{b}=0.03$, $95 \%$ CI $[0.01,0.06]$ ), and the effect of sensory-processing sensitivity via resilience on COVID-19 stress was as close to zero as possible. Thus, we can conclude that the effect of sensory-processing sensitivity on COVID19 distress is miniscule when mediated by resilience.

\section{Discussion}

Previous studies have pointed out that the COVID-19 outbreak may also be a mental health crisis, especially for adolescents, but our knowledge about this is still limited. Given this background, the present study provides new evidence on the relationship between sensoryprocessing sensitivity, resilience, and COVID-19 stress in the young population during a pandemic. This study yielded three main findings. First, higher sensory-processing sensitivity was associated with higher COVID-19 stress levels, although the effect size was not large. Second, resilient adolescents reported lower COVID-19 stress levels, replicating previous studies' findings. Third, as expected, resilience buffered the negative association between sensory-processing sensitivity and COVID19 stress. These findings could be useful for parents, educators, and healthcare providers in their quest to understand and support the mental health of highly sensitive youth.

The mean value of Japanese adolescents' COVID Stress Scale scores was approximately 1.00 (i.e., not very or rarely worried). In other words, evidently, several Japanese youths who participated in this study were not very worried about the COVID-19 pandemic. There are several possible reasons for this finding. One possible explanation is that Japan has a low infection rate compared to Western countries (WHO, 2021). Another possible interpretation is that compared to their feelings at the 
Table 1

Descriptive statistics and correlation coefficients among variables.

\begin{tabular}{|c|c|c|c|c|c|c|c|c|c|}
\hline & $M$ & $S D$ & Range & Kurtosis & Skewness & 2 & 3 & 4 & 5 \\
\hline 1. HSP & 4.48 & 1.11 & $1.20-7.00$ & -0.32 & -0.06 & $-0.48^{* * *}$ & $0.25^{\text {*水水 }}$ & -0.07 & $0.17^{* * *}$ \\
\hline 2. Resilience & 2.94 & 0.85 & $1.00-5.00$ & -0.69 & -0.06 & - & $-0.23^{* * *}$ & 0.08 & -0.07 \\
\hline 3. COVID-19 stress & 0.99 & 0.52 & $0.00-3.06$ & 0.50 & 0.61 & & - & -0.05 & $0.12^{* *}$ \\
\hline 4. Age & 18.91 & 0.82 & $18.00-24.00$ & - & - & & & - & $-0.10^{*}$ \\
\hline 5. Gender & - & - & - & - & - & & & & - \\
\hline
\end{tabular}

Note. HSP = sensory-processing sensitivity; Gender was coded as $1=$ men and $2=$ women.

* $p<.05$.

** $p<.01$.

**** $p<.001$.

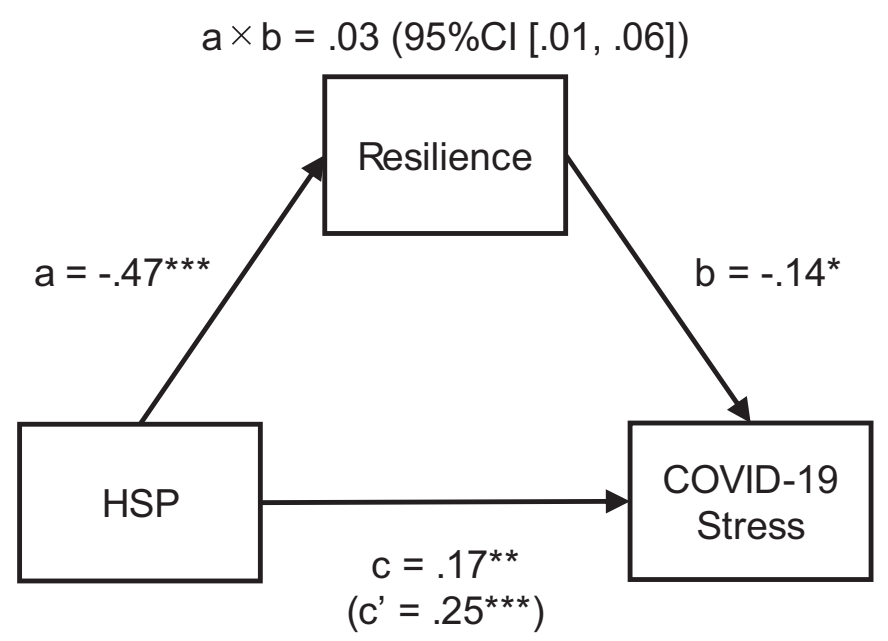

Fig. 2. Mediation model for associations between sensory-processing sensitivity, resilience, and COVID-19 stress note. This model was estimated by controlling for gender and age. HSP $=$ sensory-processing sensitivity. ${ }^{*} p<.05$, $* * p<.01, * * * p<.001$.

time of the pandemic's onset, the youth may have become accustomed to the pandemic by the time this study was conducted in October 2020. Nevertheless, approximately $3 \%$ of the sample scored an average of 2 or more on the COVID Stress Scale (i.e., somewhat or sometimes worried), and some may need support.

In this study, the mean value of the Brief Resilience Scale scores amidst the COVID-19 pandemic was 2.94. On the other hand, a prepandemic survey found that Japanese adolescents' average resilience score was 3.12 (Iimura \& Taku, 2018). Comparing the average scores across these samples, it appears that resilience (as measured by this scale) is relatively stable despite the onset of a pandemic. However, we need to examine intra-individual variability to conclude whether resilience is stable before and after a pandemic. It is worthwhile to note that approximately $16 \%$ of the Japanese youth sample had a low mean score (i.e., 1.00-2.00), suggesting that they may benefit from support aimed at increasing their resilience.

\subsection{Is sensitivity a vulnerability amidst the COVID-19 pandemic?}

Given that sensory-processing sensitivity showed a positive but small association with COVID-19 stress, high sensitivity could be a risk factor during the COVID-19 pandemic. Theories and research point to the fact that high environmental sensitivity can negatively affect youths' socioemotional development in stressful environments (Belsky \& Pluess, 2009; Monroe \& Simons, 1991). For example, Pluess et al. (2010) reported that individuals with the 5-HTTLPR s-allele, a genetic marker of environmental sensitivity, have a higher tendency toward developing the Big Five of neuroticism when they experience negative life events. In addition, Iimura (2021) indicated that when highly sensitive youths have recently experienced positive life events, there is no observable decline in their socio-emotional well-being. The key implication of these findings or theories regarding environmental sensitivity is that highly sensitive youths are not necessarily vulnerable individuals. In other words, if we can reduce negative environmental influences or increase supportive environmental influences, highly sensitive youths can have similar or even better mental health than less sensitive youths.

The mediation analysis results showed that resilience buffered the negative relationship between sensory-processing sensitivity and COVID-19 stress. Importantly, when mediated by resilience, the indirect effect of sensory-processing sensitivity to COVID-19 stress was close to zero. Given this finding, for highly sensitive youths, resilience may be an important protective factor during the COVID-19 pandemic, as previous studies using a sample of Chinese adolescents have also shown (Zhang et al., 2020). However, the present study also found that highly sensitive youths exhibited low resilience. Therefore, when considering strategies for supporting such youths, it may be important to aim to enhance resilience.

Can resilience among the youth be enhanced? Several intervention studies have demonstrated that bolstering resilience through psychoeducation and training can decrease psychopathology. For example, the SPARK resilience program (Boniwell \& Ryan, 2009), which consists of several lessons, is useful for enhancing highly sensitive youths' socioemotional well-being. Kibe et al. (2020) implemented a school-based SPARK resilience program for Japanese adolescents and reported that highly sensitive adolescents experienced decreased depression and increased self-esteem. Pluess and Boniwell (2015) also implemented this program among urban girls in the United Kingdom and saw a reduction in depressive symptoms among highly sensitive girls only. These findings suggest that highly sensitive adolescents are more likely to benefit from such interventions than less sensitive adolescents (Pluess \& Belsky, 2013).

\subsection{Limitations and implications}

To the best of our knowledge, this is the first study to report an association between COVID-19 stress and sensory-processing sensitivity in the young population. In this sense, this study is more of a hypothesisgenerating exploratory study than a hypothesis-testing one, and it has several limitations. First, as Chen and Bonanno (2020) have pointed out, although timely and informative, this study "at best, provided a snapshot of the immediate impact of COVID-19, but failed to consider the important unfolding process of psychological adjustment following stressful and potentially traumatic events" (p. S51). Following their recommendation, future research needs to examine the long-term effects of the pandemic on youth mental health. Second, the study did not control for the effects of other variables that are assumed to influence youth mental health during the COVID-19 pandemic. For example, prior research has examined a variety of factors that may be associated with psychopathology during the COVID-19 pandemic, including relationships with parents (Verdolini et al., 2021), personality traits (Zager Kocjan et al., 2021), social support (Qi et al., 2020), coping style (Zhang et al., 2020), the perceived benefits of home isolation, Internet and 
smartphone addiction (Duan et al., 2020), and online learning difficulties (Magson et al., 2021). Third, this study's findings relied on youths' self-reports. The addition of parental ratings may deepen our understanding of youths' socio-emotional well-being during the pandemic. Fourth, based on the finding that youths living in urban areas report higher psychopathology during the pandemic (Duan et al., 2020), this study collected data from urban youths in Tokyo. However, youths living in rural areas of Japan may experience different pandemic-related difficulties than their urban counterparts. Finally, as very few studies have examined the correlation between sensory-processing sensitivity and resilience (within and without the context of a pandemic), researchers should address these issues in the future to further understanding of youth mental health.

\section{Conclusion}

Researchers around the world are increasingly interested in the impact of the COVID-19 pandemic on youth mental health. The present study collected data from Japanese youths living in urban areas to examine, for the first time, whether resilience, which is known as a protective factor, buffers the relationship between the personality trait of environmental sensitivity, which has traditionally been regarded as a vulnerability factor, and COVID-19-related distress. In light of the mediation analysis results, the most important message emerging from this study is that greater sensitivity does not necessarily equate to vulnerability, if resilience can be enhanced.

\section{Funding}

This study was funded by JSPS KAKENHI Grant Numbers 19J00270 for the first author (Iimura, S.).

\section{Availability of data and material}

The data that support the findings of this study are available from the corresponding author, upon reasonable request.

\section{CRediT authorship contribution statement}

Shuhei Iimura: Conceptualization, Data curation, Formal analysis, Funding acquisition, Investigation, Methodology, Project administration, Validation, Visualization, Writing - original draft, Writing - review \& editing.

\section{Declaration of competing interest}

There is no conflict of interest with regard to this manuscript.

\section{Appendix A. Supplementary data}

Supplementary data to this article can be found online at https://doi. org/10.1016/j.paid.2021.111183.

\section{References}

Belsky, J., \& Pluess, M. (2009). Beyond diathesis stress: Differential susceptibility to environmental influences. Psychological Bulletin, 135, 885-908. https://doi.org/ $10.1037 / \mathrm{a} 0017376$.

Boniwell, I., \& Ryan, L. (2009). SPARK resilience: A teacher's guide. London, UK: University of East London.

Chen, S., \& Bonanno, G. A. (2020). Psychological adjustment during the global outbreak of COVID-19: A resilience perspective. Psychological Trauma Theory Research Practice and Policy, 12, S51-S54. https://doi.org/10.1037/tra0000685.

Duan, L., Shao, X., Wang, Y., Huang, Y., Miao, J., Yang, X., \& Zhu, G. (2020). An investigation of mental health status of children and adolescents in China during the outbreak of COVID-19. Journal of Affective Disorders, 275, 112-118. https://doi.org/ 10.1016/j.jad.2020.06.029.
Faul, F., Erdfelder, E., Buchner, A., \& Lang, A. G. (2009). Statistical power analyses using $G^{*}$ Power 3.1: Tests for correlation and regression analyses. Behavior Research Methods, 41, 1149-1160. https://doi.org/10.3758/BRM.41.4.1149.

Golberstein, E., Wen, H., \& Miller, B. F. (2020). Coronavirus disease 2019 (COVID-19) and mental health for children and adolescents. JAMA Pediatrics, 174, 819-820. https://doi.org/10.1001/jamapediatrics.2020.1456.

Greven, C. U., Lionetti, F., Booth, C., Aron, E. N., Fox, E., Schendan, H. E., ... Homberg, J. (2019). Sensory processing sensitivity in the context of environmental sensitivity: A critical review and development of research agenda. Neuroscience and Biobehavioral Reviews, 98, 287-305. https://doi.org/10.1016/j.neubiorev.2019.01.009.

Hu, L., \& Bentler, P. M. (1999). Cutoff criteria for fit indexes in covariance structure analysis: Conventional criteria versus new alternatives. Structural Equation Modeling: A Multidisciplinary Journal, 6, 1-55. https://doi.org/10.1080/10705519909540118.

Iimura, S. (2021). Highly sensitive adolescents: The relationship between weekly life events and weekly socioemotional well-being. British Journal of Psychology. https:// doi.org/10.1111/bjop.12505. Advance online publication.

Iimura, S., \& Kibe, C. (2020). Highly sensitive adolescent benefits in positive school transitions: Evidence for vantage sensitivity in Japanese high-schoolers. Developmental Psychology, 56, 1565-1581. https://doi.org/10.1037/dev0000991.

Iimura, S., \& Taku, K. (2018). Gender differences in relationship between resilience and Big Five personality traits in Japanese adolescents. Psychological Reports, 121, 920-931. https://doi.org/10.1177/0033294117741654.

Iimura, S., Yano, K., \& Ishii, Y. (2021). Environmental sensitivity in adults: Psychometric properties of the Japanese Version of the Highly Sensitive Person Scale 10-Item Version. PsyArXiv. https://doi.org/10.31234/osf.io/2h8jt.

John Hopkins University. (2020). COVID-19 dashboard by the Center for Systems Science and Engineering (CSSE) at Johns Hopkins University (JHU). Retrieved from https://coronavirus.jhu.edu/map.html.

Kibe, C., Suzuki, M., Hirano, M., \& Boniwell, I. (2020). Sensory processing sensitivity and culturally modified resilience education: Differential susceptibility in Japanese adolescents. PLoS One, 15, 1-17. https://doi.org/10.1371/journal.pone.0239002.

Killgore, W. D. S., Taylor, E. C., Cloonan, S. A., \& Dailey, N. S. (2020). Psychological resilience during the COVID-19 lockdown. Psychiatry Research, 291, Article 113216. https://doi.org/10.1016/j.psychres.2020.113216.

Liss, M., Mailloux, J., \& Erchull, M. J. (2008). The relationships between sensory processing sensitivity, alexithymia, autism, depression, and anxiety. Personality and Individual Differences, 45, 255-259. https://doi.org/10.1016/j.paid.2008.04.009.

Liss, M., Timmel, L., Baxley, K., \& Killingsworth, P. (2005). Sensory processing sensitivity and its relation to parental bonding, anxiety, and depression. Personality and Individual Differences, 39, 1429-1439. https://doi.org/10.1016/j.paid.2005.05.007.

MacKinnon, D. P., Fairchild, A. J., \& Fritz, M. S. (2007). Mediation analysis. Annual Review of Psychology, 58, 593-602. https://doi.org/10.1146/annurev. psych.58.110405.085542.

Magson, N. R., Freeman, J. Y. A., Rapee, R. M., Richardson, C. E., Oar, E. L., \& Fardouly, J. (2021). Risk and protective factors for prospective changes in adolescent mental health during the COVID-19 pandemic. Journal of Youth and Adolescence, 50, 44-57. https://doi.org/10.1007/s10964-020-01332-9.

Mertens, G., Gerritsen, L., Duijndam, S., Salemink, E., \& Engelhard, I. M. (2020). Fear of the coronavirus (COVID-19): Predictors in an online study conducted in March 2020. Journal of Anxiety Disorders, 74, Article 102258. https://doi.org/10.1016/j. janxdis. 2020.102258.

Monroe, S. M., \& Simons, A. D. (1991). Diathesis-stress theories in the context of life stress research: Implications for the depressive disorders. Psychological Bulletin, 110, 406-425. https://doi.org/10.1037/0033-2909.110.3.406.

Muta, T., Sato, H., Takashina, H., Takebayashi, Y., Yokomitsu, K., Aoki, S. Akiyama, N. (2020). The Japanese version of the COVID Stress Scale. PsyArXiv. https://doi.org/10.31219/osf.io/6apd8.

Pluess, M. (2015). Individual differences in environmental sensitivity. Child Development Perspectives, 9, 138-143. https://doi.org/10.1111/cdep.12120.

Pluess, M., \& Belsky, J. (2013). Vantage sensitivity: Individual differences in response to positive experiences. Psychological Bulletin, 139, 901-916. https://doi.org/10.1037/ a0030196.

Pluess, M., \& Boniwell, I. (2015). Sensory-processing sensitivity predicts treatment response to a school-based depression prevention program: Evidence of vantage sensitivity. Personality and Individual Differences, 82, 40-45. https://doi.org/ 10.1016/j.paid.2015.03.011.

Pluess, M., Belsky, J., Way, B. M., \& Taylor, S. E. (2010). 5-HTTLPR moderates effects of current life events on neuroticism: differential susceptibility to environmental influences. Progress in Neuro-Psychopharmacology \& Biological Psychiatry, 34(6), 1070-1074. https://doi.org/10.1016/j.pnpbp.2010.05.028.

Prime, H., Wade, M., \& Browne, D. T. (2020). Risk and resilience in family well-being during the COVID-19 pandemic. The American Psychologist, 75(5), 631-643. https:// doi.org/10.1037/amp0000660.

Qi, M., Zhou, S.-J., Guo, Z.-C., Zhang, L.-G., Min, H.-J., Li, X.-M., \& Chen, J.-X. (2020). The effect of social support on mental health in Chinese adolescents during the outbreak of COVID-19. Journal of Adolescent Health Care, 67, 514-518. https://doi. org/10.1016/j.jadohealth.2020.07.001.

R Core Team. (2020). R: A language and environment for statistical computing. Vienna, Austria: R Foundation for Statistical Computing. Retrieved from https://www.R-pro ject.org/.

Sawyer, S. M., Azzopardi, P. S., Wickremarathne, D., \& Patton, G. C. (2018). The age of adolescence. The Lancet Child \& Adolescent Health, 2, 223-228. https://doi.org/ 10.1016/S2352-4642(18)30022-1.

Schermelleh-Engel, K., Moosbrugger, H., \& Müller, H. (2003). Evaluating the fit of structural equation models: Tests of significance and descriptive goodness-of-fit measures. Methods of Psychological Research Online, 8, 23-74. 
Smith, B. W., Dalen, J., Wiggins, K., Tooley, E., Christopher, P., \& Bernard, J. (2008). The brief resilience scale: assessing the ability to bounce back. International Journal of Behavioral Medicine, 15(3), 194-200. https://doi.org/10.1080/ 10705500802222972.

Tang, S., Xiang, M., Cheung, T., \& Xiang, Y.-T. (2021). Mental health and its correlates among children and adolescents during COVID-19 school closure: The importance of parent-child discussion. Journal of Affective Disorders, 279, 353-360. https://doi.org/ 10.1016/j.jad.2020.10.016.

Taylor, S., Landry, C. A., Paluszek, M. M., Fergus, T. A., McKay, D., \& Asmundson, G. J. G. (2020). Development and initial validation of the COVID Stress Scales. Journal of Anxiety Disorders, 72, Article 102232. https://doi.org/10.1016/j. janxdis.2020.102232.

Tokuyoshi, Y., \& Moriya, M. (2014, September). Development and validation of the Brief Resilience Scale Japanese version (BRS-J). In Poster session presented at the annual convention of the Japanese Psychological Association, Nagoya Congress Center, Nagoya, Japan.

van der Velden, P. G., Contino, C., Das, M., van Loon, P., \& Bosmans, M. W. G. (2020) Anxiety and depression symptoms, and lack of emotional support among the genera population before and during the COVID-19 pandemic. A prospective national study on prevalence and risk factors. Journal of Affective Disorders, 277, 540-548. https:// doi.org/10.1016/j.jad.2020.08.026.

Verdolini, N., Amoretti, S., Montejo, L., García-Rizo, C., Hogg, B., Mezquida, G., ... Solé, B. (2021). Resilience and mental health during the COVID-19 pandemic. Journal of Affective Disorders, 283, 156-164. https://doi.org/10.1016/j. jad.2021.01.055.

World Health Organization. (2021). WHO coronavirus (COVID-19) dashboard. Retrieved from https://covid19.who.int/.

Xiong, J., Lipsitz, O., Nasri, F., Lui, L. M. W., Gill, H., Phan, L., ... McIntyre, R. S. (2020). Impact of COVID-19 pandemic on mental health in the general population: A systematic review. Journal of Affective Disorders, 277, 55-64. https://doi.org/ 10.1016/j.jad.2020.08.001.

Zager Kocjan, G., Kavčič, T., \& Avsec, A. (2021). Resilience matters: Explaining the association between personality and psychological functioning during the COVID-19 pandemic. International Journal of Clinical and Health Psychology, 21, Article 100198. https://doi.org/10.1016/j.ijchp.2020.08.002.

Zhang, C., Ye, M., Fu, Y., Yang, M., Luo, F., Yuan, J., \& Tao, Q. (2020). The psychological impact of the COVID-19 pandemic on teenagers in China. The Journal of Adolescent Health, 67, 747-755. https://doi.org/10.1016/j.jadohealth.2020.08.026. 